\title{
Identifying student resources for understanding linear momentum
}

\author{
Brynna Hansen \\ Department of Biology, Seattle Pacific University, 3307 Third Ave W, Seattle, WA, USA, 98119-1997 \\ Lauren C. Bauman \\ Department of Physics, University of Washington, Box 351560, Seattle, WA, USA, 98195-15603 \\ Yohannes M. Abraham \\ Department of Biology, Seattle Pacific University, 3307 Third Ave W, Seattle, WA, USA, 98119-1997 \\ Mikayla Valentin and Amy D. Robertson \\ Department of Physics, Seattle Pacific University, 3307 Third Ave W, Seattle, WA, USA, 98119-1997
}

Much existing physics education research (PER) on student ideas about momentum focuses on the difficulties that students face when learning this topic. These difficulties are framed as obstacles for students to overcome in order to develop correct understandings of physics. Our research takes a resources-oriented approach to analyzing student responses to momentum questions, viewing student ideas as valuable and potentially productive for learning, over and above their correctness. Here, we highlight four conceptual resources that provide insight into students' ideas about momentum, which are the conservation resource, direction resource, collisions resource and properties resource. These resources are context-dependent and could be elicited and built on by instructors to support students in developing more complex and sophisticated understandings of physics.

\footnotetext{
2021 PERC Proceedings edited by Bennett, Frank, and Vieyra; Peer-reviewed, doi.org/10.1119/perc.2021.pr.Hansen

Published by the American Association of Physics Teachers under a Creative Commons Attribution 4.0 license.

Further distribution must maintain the cover page and attribution to the article's authors.
} 


\section{INTRODUCTION}

Physics education research (PER) has contributed extensively to instructors' pedagogical content knowledge (PCK), or specialized knowledge for teaching, which includes both an understanding of the content being taught and knowledge of student ideas (KSI) [1]. Previous research on student ideas about linear momentum has primarily been misconceptions-oriented, contributing to KSI by identifying student misconceptions or difficulties in learning about momentum. For example, the literature reports that students: (1) tend to treat momentum as a scalar quantity [2-7], (2) think about momentum as dependent on either velocity or mass, but not both [2-4,7-12]; and (3) misunderstand or have difficulty applying conservation reasoning, in some cases using "compensation reasoning," treating the velocity of an object as though it "adjusts" to maintain a constant momentum [3,5-7,12-15].

Our research aims to contribute to instructors' KSI by identifying student resources, which are ideas or patterns of reasoning that could represent the beginnings of more sophisticated understandings of physics [16-19]. In contrast to misconceptions-oriented research, which identifies student reasoning that is discontinuous with formal physics reasoning, our work identifies resources as elements of student thinking that are or could be continuous with formal physics, regardless of the correctness of student responses.

We asked the question: What are some of the common conceptual resources that students use to answer linear momentum questions? We analyzed 656 student responses to three conceptual questions about linear momentum. We identified four resources for understanding momentum: A) the conservation resource, B) the direction resource, C) the collisions resource, and D) the properties resource. Our work seeks to add to instructors' PCK by making visible the continuities between students' thinking and canonical physics, even and especially when that thinking does not use the language of formal physics or is incorrect.

\section{THEORETICAL FRAMEWORK}

In resources theory, a resource is a piece of knowledge that is activated in real time, in context-sensitive ways to form an idea, explanation, argument, or theory $[16,17,19$ 25]. Researchers have theorized extensively about the development, structure, and role of resources, and have used resources theory to highlight the dynamic, emergent, complex-systems-like nature of student thinking [19].

Our work draws extensively from resource theory's orientation toward student thinking as fundamentally sensible and continuous with formal physics [16,19$22,24,25]$. Resource theory emphasizes that learners derive resources from their physical or sensory experience and then use these resources to make sense of the material world. Further, our work builds from resource theory's definition of learning, which involves changing the structure or activation of resources, by reorganizing, refining, increasing the degree of formality of, or changing the role of resources $[16,17,20,21,24,25]$. Our primary aim in identifying resources is to provide instructors with knowledge that they can use to build from student ideas in instruction. Finally, our work assumes that resource activation is contextsensitive $[16,17,19,21,22,24,25]$ and hypothesizes aspects of context that may shape which resources get activated. Many of the resources we identify are at the grain size of an idea (rather than, for example, a primitive), and this is not inconsistent with resources theory [16].

\section{METHODS}

The resources we report in this paper were identified in written student responses to three momentum questions: the shut-the-door question, the desk-chair question, and the sticky blocks question (Figure 1). The desk-chair and sticky blocks questions were based on previous literature [5,26]. The shut-the-door question was constructed for this project.

In the shut-the-door question, with the door initially at rest, the initial momentum of the system is the momentum of the clay/ball. If we assume these initial momenta are the same (e.g., we throw them with the same speed in the same direction and they are equally massive), then the vector representing the momentum of the system points in the same direction and is the same length for both scenarios. The clay sticks to the door and continues to have a component of motion in its original direction, and the rubber ball bounces and moves in the opposite direction. By vector addition, the final momentum of the door (which moves in ball's original direction of motion) would be higher with the rubber ball. For the desk-chair question, part (a), we can make sense of the catcher's movement in terms of momentum transfer: when the ball collides with the person, it gives some of its momentum to the person, and they begin to move. The ball and the person move more slowly than the ball did originally because of momentum conservation: when the ball gives some momentum to the person, it moves slower. The person is presumably more massive than the ball and receives only a part of the ball's momentum so also moves slower than the ball did. For part (b), the original (vector) momentum of the system was zero. When the ball moves one way, the thrower must move the other way to conserve momentum. In the sticky-blocks question, the initial momentum of the system of the two blocks is zero, since they are equally massive and moving in opposite directions. The final momentum and thus the system's velocity must also then be zero. If the blocks are stuck together, and the system's velocity is zero, the blocks are at rest.

We analyzed a total of 656 written responses from introductory physics courses at five US colleges and universities. U1, U2, U3, and $\mathrm{U} 4$ are all large public institutions, U1 and U2 in the Pacific Northwest US, U3 in the Eastern US, and U4 in the Midwest. U5 is a small private 


\begin{tabular}{|c|c|c|}
\hline $\begin{array}{l}\text { Shut-the-door question (Q1): } \\
\text { Imagine you want to shut a door } \\
\text { quickly but can't reach it. You have } \\
\text { at your disposal a rubber bouncy ball } \\
\text { and an equally massive piece of clay. } \\
\text { Which of these would you choose to } \\
\text { throw at the door so that you have the } \\
\text { higher chance to shut the door } \\
\text { quickly and why? }\end{array}$ & $\begin{array}{l}\text { Sticky-blocks question (Q2): } \\
\text { Two identical blocks of inertia } m \text { slide towards each } \\
\text { other at equal speed } v \text { on a frictionless surface, as in } \\
\text { the picture at right. The blocks are sticky on the sides } \\
\text { not touching the floor, and they stick together when } \\
\text { they collide. } \\
\text { After the collision, how } \\
\text { fast do the blocks } \\
\text { moves, and in what } \\
\text { direction? Explain why } \\
\text { your answer makes } \\
\text { sense to you. }\end{array}$ & $\begin{array}{l}\text { Desk-chair question (Q3): } \\
\text { Two students are facing one another while sitting at rest in } \\
\text { desk chairs with low-friction bearing on a slippery surface. } \\
\text { One of the students tosses a large, heavy ball to the other. } \\
\text { (a) The student who catches the ball begins to move in the } \\
\text { direction that the ball was originally moving. Both the ball } \\
\text { and the student move more slowly than the ball was } \\
\text { moving just before the student caught it. Why does this } \\
\text { make sense to you? (If it doesn't make sense, say why not.) } \\
\text { (b) The student who tosses the ball begins moving in the } \\
\text { opposite direction of the ball's motion. Why does this make } \\
\text { sense to you? (Again, if it doesn't make sense, say why } \\
\text { not.) }\end{array}$ \\
\hline
\end{tabular}

FIG. 1. The conceptual questions asked in our study.

institution in the Pacific Northwest. (Sample sizes by question and college/university are given in Table I.) Students answered the questions on homework and quizzes, before and after instruction on linear momentum. The course response rates were $85 \%-94 \%$ for questions asked at U1; $68 \%-83 \%$ for $\mathrm{U} 2 ; 53 \%$ for $\mathrm{U} 3 ; 33 \% \mathrm{U} 4$, and $74 \%$ for $\mathrm{U} 5$. Lower course response rates correspond to data collected: at the start of the global COVID-19 pandemic, from group assignments, and in courses with fewer students consenting to participate in research.

The racial and/or ethnic demographics for the colleges/ universities in our study versus all college/university students are shown in Figure 2. Figure 2 suggests that the institutions in our study are not racially and/or ethnically representative of the population of college-bound freshmen in the US. The universities in our study serve more Asian and Asian American students, fewer Hispanic or Latinx students, fewer Black or African American students, and fewer white students than are in the general population of college students. In addition, the median parental income of the students at colleges/universities in our study is higher than the national average. This sampling limits the generalizability of our results; though we have documented that the resources we have identified are common among the students in our sample, we cannot speak to their commonality in the population of introductory physics students writ large. One limitation of Figure 2 and our comparison of wealth demographics is that they are based on university-level data, rather than data at the level of our sample. We are accumulating sample-level data, but until we know more about what constitutes a representative sample of introductory physics students, sample-level data does not allow for comparisons to a national average. We used responses to the questions in Figure 1 to create an emergent coding scheme [27] to capture some common resources that the students in our sample were using to reason about linear momentum. To construct this scheme, authors BH and LB, in consultation with $\mathrm{AR}, \mathrm{YA}$, and $\mathrm{MV}$, conducted preliminary analyses of student responses to each question, looking for ideas that we considered to be continuous with relevant formal physics concepts, even if not stated in formal

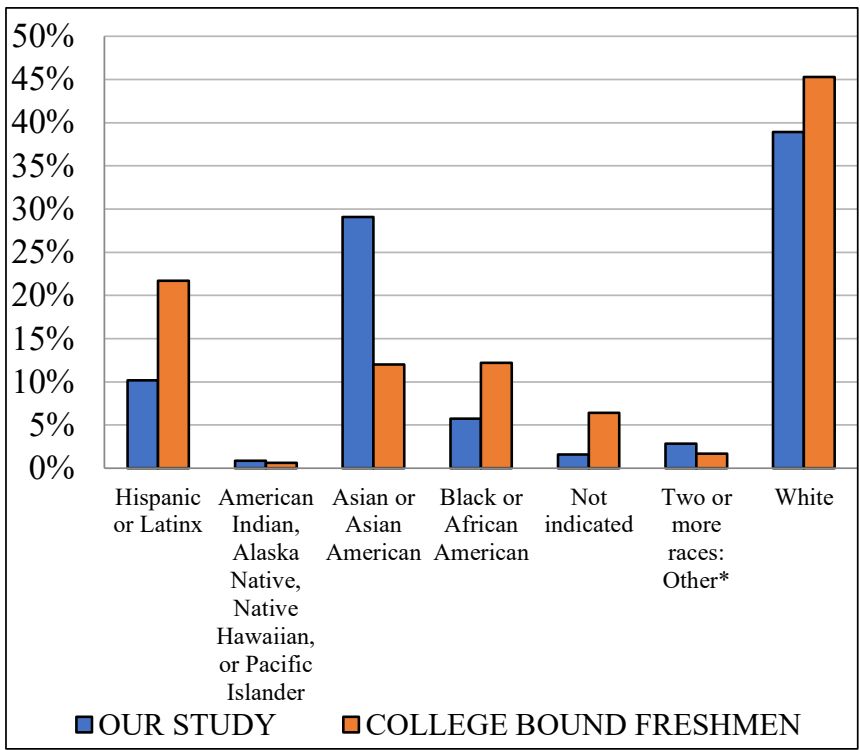

FIG. 2. Racial and/or ethnic demographics of institutions in our sample (blue) versus all college-bound freshmen (orange). Blue bars were constructed using demographic data provided by offices of institutional research or institutional websites, weighted by sample size. Orange bars were constructed using data from Kanim and Cid [28]. As explained by activist Kat Lazo [29], neither Hispanic nor Latinx are racial groups, and these two identities are not the same. "Hispanic" is a descriptor for people of Spanishspeaking origins, and "Latinx" is a descriptor for people with origins in Latin America. The former focuses on language, the latter on geographic location.

terms. We then identified patterns across questions, producing a final scheme with four resources. This approach of pattern-seeking foregrounds a model of generalizability that emphasizes recurrence across multiple sources of heterogeneity [30].

Authors BH and LB then independently coded student responses to the three questions. Resources can be and often are activated in concert; thus, a single response could receive no code, one code, or many codes. As a measure of interrater agreement, we took the normalized difference between the total number of possible codes and the total number of disagreements between the two coders. We used percentage 
agreement rather than a standard statistical measure of agreement (e.g., Cohen's kappa) because our codes are not independent or mutually exclusive $[31,32]$. The percentage agreement for the full data set was $89 \%$. Disagreements were not resolved through discussion; instead the percentages reported in Table I represent the fraction of responses for which both authors were in agreement.

\section{RESULTS}

In this section we highlight four resources for understanding linear momentum that were common in student responses in our sample. We considered the resources to be "common" if they appeared in at least $10 \%$ of student responses to at least one question, and also appeared at some frequency in response to multiple questions. Table I lists the prevalence of each resource in the three questions we asked, across the sampled institutions.

TABLE I. Prevalence of each resource by university and question. U1 asked students all three conceptual questions (Q1-3), U2 asked Q1 and Q3, U3 asked Q2, and U4 and U5 asked Q3. Percentages represent responses for which both coders agreed.

\begin{tabular}{lll|ll|llll}
\hline \multicolumn{1}{c|}{} & \multicolumn{9}{|c}{ Prevalence } \\
\hline & & Q1 & \multicolumn{3}{|c}{ Q2 } & \multicolumn{3}{c}{ Q3 } \\
& $\mathrm{U} 1$ & $\mathrm{U} 2$ & $\mathrm{U} 1$ & $\mathrm{U} 3$ & $\mathrm{U} 1$ & $\mathrm{U} 2$ & $\mathrm{U} 4$ & $\mathrm{U} 5$ \\
$n$ & 163 & 58 & 163 & 42 & 56 & 122 & 18 & 34 \\
\hline $\mathrm{A}$ & $24 \%$ & $9 \%$ & $45 \%$ & $24 \%$ & $59 \%$ & $30 \%$ & $0 \%$ & $41 \%$ \\
$\mathrm{~B}$ & $10 \%$ & $2 \%$ & $26 \%$ & $10 \%$ & $16 \%$ & $10 \%$ & $0 \%$ & $12 \%$ \\
$\mathrm{C}$ & $34 \%$ & $28 \%$ & $18 \%$ & $12 \%$ & $5 \%$ & $3 \%$ & $0 \%$ & $0 \%$ \\
$\mathrm{D}$ & $6 \%$ & $22 \%$ & $5 \%$ & $10 \%$ & $0 \%$ & $2 \%$ & $0 \%$ & $3 \%$ \\
\hline
\end{tabular}

\section{A. "Conservation" Resource}

Table I shows that between $9 \%$ and $59 \%$ of student responses used the conservation resource, depending on the question and sample, and that this resource was common in responses to all three conceptual questions. Student responses that drew on the conservation resource used the conservation and/or transfer of momentum to explain or predict the motion of objects after a collision. In these cases, students described conservation as a governing factor of an object's behavior during and following a collision. Some answers connected conservation and transfer, stating or implying that the transfer of momentum is the mechanism by which momentum is conserved in a system. The use of this resource is consistent with the canonical view of momentum as a quantity that must be conserved in isolated systems.

An example of this resource comes from a student response to the desk-chair question: "This makes sense because after the collision, some of the momentum of the ball transfers to the catcher, causing the catcher to start moving and the ball to move at a slower velocity." This student notes that momentum from the tossed ball is transferred to the catcher, which makes the catcher move from rest and causes the ball to move at a slower velocity.
While not explicitly stated by the student, conservation of momentum is implied: the transfer of momentum meant the ball moves slower (and has less).

\section{B. "Direction" Resource}

Between $2 \%$ and $26 \%$ of student responses used the direction resource, depending on the question and sample, as shown in Table 1. The direction resource foregrounds that momentum is the kind of quantity that has a direction and the importance of directionality for momentum calculations. Responses of this kind are consistent with the canonical definition of momentum as a vector. We identified this resource in student responses that either described momentum as being positive or negative, or used momentum vectors to map out the interactions of objects in a collision, usually through equations or diagrams.

For example, in the sticky-blocks question, one student wrote: "After the collision the blocks are at rest. Since $\mathrm{Mi}_{1} \mathrm{Vi}_{1}+\mathrm{Mi}_{2} \mathrm{Vi}_{2}=\mathrm{Mf}_{1} \mathrm{Vf}_{1}+\mathrm{Mf}_{2} \mathrm{Vf}_{2}$ and one object is moving in the negative direction, their momentums will cancel each other out and equal zero. Since they are identical and moving at the same speed. For the final momentum to equal zero, the velocity will also have to equal zero, so the blocks are at rest." In this response, the student uses the direction of the blocks' movement to determine that the blocks' momenta will "cancel each other out" and that the final momentum will need to equal zero.

Another student justified choosing the bouncy ball in the shut-the-door question, stating: "The momentum when it approaches the door is positive. Assuming this happens in a closed system, momentum is conserved. So, when the bouncy ball hits the door, it moves in a negative direction \& has a negative momentum, causing the door to have a positive momentum in the opposite direction." This response illustrates attention to the directionality of momentum at different moments during and after the collision. Using the "direction" resource in concert with the conservation resource, this student predicts the motion of door based on the "positive" and "negative" direction of the momentum.

\section{C. "Collision" Resource}

We identified the collision resource in between $3 \%$ and $34 \%$ of responses; this resource was most prevalent in responses to the shut-the-door question. Students using the collision resource implied that the type of collision that an object undergoes, such as elastic or inelastic, matters for its momentum, formally or informally applying a constraint to their analysis of the system.

For example, in response to the shut-the-door predict question, one student wrote: "I would choose the bouncy ball because it's going to bounce off the door with close to similar momentum. $\mathrm{p}=(\mathrm{mv}--\mathrm{mv})=2 \mathrm{mv}$. Since it bounces off the door with close to similar momentum the change in momentum is double causing the force ( $\mathrm{f}=\mathrm{ma}$ ) to increase as momentum and force are proportional according to $\mathrm{a}=\mathrm{vt}$ 
and mass and time are constant." In this response, the student describes an elastic collision between the bouncy ball and door, noting that because the ball bounces off the door (changes direction), its change in momentum is double. In the context of the shut-the-door question, this resource captures the idea that if the result is different for the bouncy ball and the clay, it will also be different for the door. In other words, the outcome of the collision is influenced by the interactions that occur during the collision.

\section{D. "Properties" Resource}

The properties resource was used in between $2 \%$ and $22 \%$ of responses and was most prevalent in answers to the shut-the-door question. Student responses used this resource when they suggested that the properties of an object matter for how they interact with other objects in a collision, and for the resulting momentum of the objects. The structure of the argument was often: because the objects that collided have a certain property (hard, soft, bouncy, heavy, light, fast, etc.), their momentum changes in a certain way. This resource is similar to the collision resource, but rather than focusing on the type of collision, students focus on the properties of the object.

In response to the shut-the-door question, one student wrote: "The [motion of the door will be the] same. When they [rubber ball and clay] collide the door, the momentums of the door change in the same magnitude because they have the same mass." This student reasons that since the rubber ball and clay are equally massive, the door they hit will experience equal changes in the magnitudes of momentum. While this response is incorrect, the student is both (i) considering a property of the objects (mass) that are relevant to momentum and (ii) recognizing that the properties of the object matter for what happens in a collision between them.

The collisions and properties resources are two different means of applying constraints beyond conservation, to draw specific conclusions about the momentum of objects in a system after a collision. Students use information about the type of collision in the collisions resource and information about the properties of the object(s) in the properties resource. In some cases, students reasoned that an object's properties, or the type of collision it is involved in, determine the amount of momentum that it has, or transfers, to another object in a collision. Students often reasoned that since change in momentum and force are related, an object with a greater change in momentum will exert more force in a given amount of time. This reasoning is consistent with diSessa's characterization of momentum as a carrier of force [33].

\section{DISCUSSION}

In this paper, we offer a preliminary answer to the question, "What are some of the common conceptual resources that students use to answer momentum questions?" by identifying four resources that were common among the 656 student responses we analyzed. Our work reframes and offers additional context for some of the difficulties reported in the literature and highlights resources that to our knowledge have not been reported in prior work, offering additional KSI to physics instructors.

In particular, our work offers a reframing of literature citing student difficulties in understanding and applying conservation of momentum. Though some student responses that used the conservation resource were consistent with treating momentum as a constant [14], our work emphasizes the continuity of such responses with momentum conservation. That is, we frame student reasoning as a starting point (not a barrier): students are naming momentum as the kind of thing that does not just go away or disappear.

Further, student responses that used the conservation resource sometimes treated momentum as substance-like, in referring to it as a quantity that can move from one location or object to another and is conserved, consistent with Reiner et al's claim that students' conceptions of physics are shaped in part by substance-like metaphors, which are drawn from their experiences of the physical world [34]. Student responses to our questions often illustrated the affordances of this model: students drew on a substance metaphor to reason about momentum conservation and transfer, something we want to see happening.

Previous literature has also noted that students often treat momentum as a scalar $[2,3,7]$ rather than a vector quantity. Our data suggest that a fraction of students in fact treat momentum as a vector quantity and consider direction an important factor in the exchange of momentum among objects. This raises questions about the context-dependence of the direction resource and its activation: perhaps our questions are more likely to cue the direction resource, and questions from previous studies are more likely to cue scalar notions of momentum. To our knowledge, the literature does not report categories of student reasoning akin to the collision and properties resources.

The context-dependence of resource activation is welldocumented [17, 24] and this bears out in our study. Table I illustrates that the four resources appear at varying frequencies in the three different questions that we asked. With our preliminary research we can only speculate as to why this may be the case. For example, that the collision and properties resources are most prevalent in responses to the shut-the-door question makes sense, as students were comparing the outcomes of two different collisions, where the salient difference is in the properties of the incoming objects. The ways in which population variability may change results like these is an open question [28].

\section{ACKNOWLEDGMENTS}

This work was supported in part by the National Science Foundation under grant numbers $1914603 \& 1914572$. The authors wish to thank Lisa Goodhew, Paula Heron, and Rachel Scherr for feedback on drafts of the paper, and all those who helped collect data. 
[1] L. Shulman, Harv. Ed. Rev. 57, (1987).

[2] H. G. Close and P. R. L. Heron, Am. J. Phys. 78, 961 (2010).

[3] T. Graham and J. Berry, Int. J. Sci. Ed.18, 75 (1996).

[4] A. G. Ç. Şekercioğlu and M. S. Kocakülah, J. Turkish Sci. Ed. 5, 2 (2008).

[5] C. Singh and D. Rosengrant, Am. J. Phys. 71, 607 (2003).

[6] C. Singh and D. Rosengran, in Proceedings of Physics Education Research Conference (2001).

[7] G. Triyani, A. Danawan, I. Suyana, and I. Kaniawati, J. Phys.: Conf. Ser. 1280, 052008 (2019).

[8] S. A. Amalia, E. Suhendi, I. Kaniawati, A. Samsudin, N. J. Fratiwi, S. R. Hidayat, A. Zulfikar, F. N. Sholihat, D. S. Jubaedah, A. H. Setyadin, M. G. Purwanto, M. H. Muhaimin, S. S. Bhakti, and N. F. Afif,, J. Phys.: Conf. Ser. 1204, 012073 (2019).

[9] T. G. K. Bryce and K. MacMillan, J. Res. Sci. Teach. 46, 739 (2009).

[10] O. Chittasirinuwat, T. Kruatong, and B. Paosawatyanyong, AIP Conference Proceedings 1263, 79 (2010).

[11] N. E.-H. Diyanahesa, S. Kusairi, and E. Latifah, J. Phys.: Theories and App. 1, 2 (2017).

[12] N. Grimellini-Tomasini, B. Pecori-Balandi, J. L. Pacca, and A. Villani, Sci. Ed. 77, 169 (1993).

[13] H. G. Close and P. R. L. Heron, Am. J. Phys. 79, 1068 (2011).

[14] R. A. Lawson and L. C. McDermott, Am. J. Phys. 55, 811 (1987).

[15] T. O. Pride, S. Vokos, and L. C. McDermott, Am. J. Phys. 66, 147 (1998).

[16] D. Hammer, Am. J. Phys. 68, S52 (2000).

[17] D. Hammer, A. Elby, R. E. Scherr, and E. F. Redish, in Transfer of Learning from a Modern Multidisciplinary Perspective (IAP, Greenwhich, CT, 2005), pp. 89-119.

[18] D. Hammer and E. van Zee, Seeing the Science in Children's Thinking: Case Studies of Student Inquiry in Physical Science, 1st edition (Heinemann, Portsmouth, NH, 2006).

[19] D. Brown and D. Hammer, in International Handbook of Research on Conceptual Change, edited by S. Vosniadou (Taylor and Francis, Inc., 2008), pp. 127154.

[20] J. P. Smith, A. A. diSessa, and J. Roschelle, J. the Learn. Sci. 3, 115 (1993).

[21] A. A. diSessa, Cog. Instruc. 10, 105 (1993).

[22] B. Harrer, Identifying Productive Resources in Secondary School Students' Discourse About Energy, (2013).

[23] R. E. Scherr, Am. J. Phys. 75, 272 (2007).

[24] E. Redish, A Theoretical Framework for Physics Education Research: Modeling Student Thinking, (2004).

[25] D. Hammer, J. Learn. Sci. 5, 97 (1996).
[26] R. E. Scherr and A. Elby, Maryland Open Source Tutorials in Physics Sensemaking.

[27] K. H. Krippendorff, Content Analysis - 3rd Edition: An Introduction to Its Methodology (SAGE Publications, Inc, Thousand Oaks, 2013).

[28] S. Kanim and X. C. Cid, Phys. Rev. Phys. Educ. Res. 16, 020106 (2020).

[29] V.M.Massie, Retrieved from: https://www.vox.com/2016/8/28/12658908/latinohispanic-race-ethnicity-explained.

[30] T. D. Cook, Educ. Eval. Pol. Analy. 24, 175 (2002).

[31] M. L. McHugh, Biochem Med. 22, 276 (2012).

[32] J. Cohen, Educ. Psych. Meas. 20, 37 (1960).

[33] A. A. diSessa, Am. J. Phys. 48, 365 (1980).

[34] M. Reiner, J. D. Slotta , M. T. H. Chi \& L. B. Resnick (2000) Cognition Instruct. 18, 1 (2000). 